UNITED STATES DEPARTMENT OF THE INTERIOR

GEOLOGICAL SURVEY

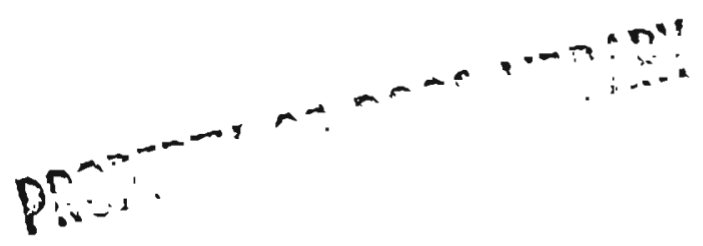

METALLIC MINERAL RESOURCES MAP OF THE MOUNT FAIRWEATHER QUADRANGLE, ALASKA

Complied by

Edward H. Cobb

Open-rile map

1968

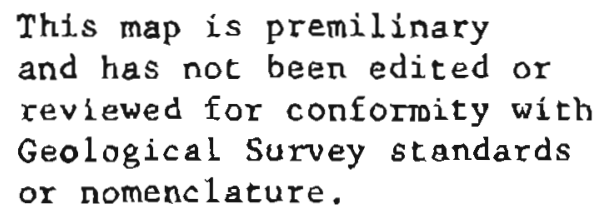


Numbex Name and principal reference(s)

1 Lituya Bay: Kennedy and Walton (1946), P. 71

South Crillon Glacier: Kennedy and Walton (1946), P. 71; Mackevett and others (1967), P. 90, 133

North Crillon Glacier: Kennedy and Walton (1946), p, 71

North Crillon Glacier: Mackevett and others (1967), p. 132

North Crillon Glacier: Mackevett and others (1967), p. 89

Mount Marchafnville: Mackevett and others (1967), p. 89

Brady Glacier: Mackevett and others (1967), p. 2, 6-7, $134,153-158$

Astrolabe Peninsula: Rossman (1963a), p. F44-F45

Johns Hopkins In1et: Mackevett and others (1967), p. 98

Johns Hopkins Inlet: Mackevett and others (1967), p. 86-87

Tarr Inlet: Mackevett and others (1967), p. 86

Tarr Inlet: Mackevett and others (1967), P. 86

Hoonah Glacier: Mackevert and others (1967), P. 89

Johns Hopkins Inlet: Mackevett and

other

Mount Cooper: Mackevetr and others (1967), p. 98

Richtmeyer: Mackevett and ochers (1967), p. 101

Parker: Twenhofel, Reed, and Gates (1949), P. 33-34

$\mathrm{Cu}$

$\mathrm{Cu}$

$\mathrm{Cu}$

Unnamed occurrence: Mackevett and others (1967), p. 133 Fe

$\mathrm{Cr}, \mathrm{Co}, \mathrm{Cu}, \mathrm{Ni}$

$\mathrm{Fe}$

$\mathrm{Pb}, \mathrm{Mo}, \mathrm{Zn}$

$\mathrm{Sb}, \mathrm{Cu}, \mathrm{Mo}, \mathrm{Ag}$

$\mathrm{Cu}, \mathrm{Ag}, \mathrm{Zn}$

$\mathrm{Cu}$

Johns Hopkins Inlet: Mackevetc and others (1967), p. 151 Mo

Cu, MO

$(1967), P .87$

$\mathrm{Cu}, \mathrm{Mo}$

Mo, Zn

Au

$\mathrm{Au}$

Symbols - Sb, antimony; $\mathrm{Cr}$, ctromite; Co, cobalt; $\mathrm{Cu}$, copper; $\mathrm{Au}$, gold; Fe, iron; $\mathrm{Pb}$, lead; Mo, molybdenum; $\mathrm{Ni}$, nickel; $\mathrm{Ag}$, silver, $\mathrm{Zn}$, zinc. 
Number Name and principal reference(s)

Commodity $1 /, \underline{2} /$

21 LeRoy: Twenhofel, Reed, and Gates (1949), p. 32-34; Rossman (1959a), P. 45-46; Mackevett and others (1967), P. $101-104$

$\mathrm{Cd}, \mathrm{Cu}, \underline{\mathrm{Au}}, \mathrm{Pb}, \mathrm{Ag}$, $\mathrm{Z} n$

$\mathrm{Zn}$

$\underline{\mathrm{Au}}, \mathrm{Pb}$

24 Rainbow: Twenhofe1, Reed, and Gates (1949), P. 31, 33-34; Mackevett and others (1967), p. 105-106

Au, $\mathrm{Pb}, \mathrm{Ag}, \mathrm{Zn}$

$\underline{\mathrm{Au}}, \mathrm{Pb}$ (1967), p. 106

26 Incas: Rossman (1959a), P. 46-48; Mackevert and others (1967), P. 108-109

27 Rambler: Rossman (1959a), P. 55; Mackevett and others (1967), P. 112

28 Highland Chief: Rossman (1959a), P. 54-55

29 Galena: Twenhofel, Reed, and Gates (1949), p. 31, 33: Mackevett and others (1967), P. 110

30 Sunrise: Rossman (1959a), p. 38; Mackevett and others (1967), p. 109

31 Hopalong: Rossman (1959a), P. 56

Whirlaway: Rossman (1959a), p. 56

Unnamed Occurrence: Rossman (1959a), p. 56

32 Lamplugh Glacier: Mackevett and others (1967), P. 88

33 Lamplugh Glacier: Mackevett and others (1967), P. 152 N1

34-35 Reld Glacier: Mackevett and others (1967), p. 151 Mo

36 Reid Glacier: Mackevett and others (1967), p. $88 \quad \mathrm{Cu}$

37 Russel1 Island: Mackevett and others (1967), P. $118 \quad \mathrm{Au}, \mathrm{Pb}, \mathrm{Ag}$

l/ Symbols - Cd, cadrolum; Cu, copper; Au, gold; Pb, lead; Mo, molybdenum; Ni, nlckel; Ag, silver; W, tungsten; $\mathrm{Zn}, \mathrm{zlnc}$.

2/ Symbol underlined Indicates recorded production.
$\mathrm{Au}, \mathrm{Pb}, \mathrm{Zn}$

$\mathrm{Au}$

Au, $\mathrm{Pb}, \mathrm{Ag}, \mathrm{Zn}$

$\mathrm{Au}, \mathrm{Ag}, \mathrm{W}$

$\frac{\mathrm{Au}}{\mathrm{Au}}$

$\mathrm{Cu}, \mathrm{Mo}$

1

Mo

u 
Number Name and princlpal reference(s)

38

39

40

41

42

43

44

45

46

47

48

49

50

51

52

53

54

55

56

I/ others (1967), D. 56, 126 others (1967), p. 83-84 others $(1967), \mathrm{p}, 57,82-83$ (1967), P. 149 others (1967), p. 79-81 152

P. $138-148$
Rendu Inlet: Mackevet and others (1967), P. 150

Rendu Inlet: Rossman (1963b), P. K48-K49; Mackevett and

Rendu Inlet: Mackevett and others (1967), p. 128-129

Rendu Inlet: Mackevett and others (1967), p. 82

Gilbert Island: Mackevett and others (1967), P. 163

Gilbere Island: Rossman (1963b), P. K49

Hugh Miller Inlet: MacKevett and others (1967), p.97

Gilbert Island: Rossman (1963b), p. K50; Mackevett and

Unnamed island: Mackevett and others (1967), P. 83-84

Blue Mouse Cove: Rossman (1963b), p. K50; Mackevett and

Triangle Island: Rossman (1963b), P. K49

Queen Inlet: Mackevett and others (1967), P. 129-131

Wachusett Inlet (Plateau Glacier): Mackevett and others

Bruce Hills: Rossman (19636), P. K49-K50; Mackevett and

Tidal Inlet: Mackevert and others (1967), P. 82

Curtls Hills: Mackevett and others (1967), P. 69-70, 134,

Nunatak: Twenhofel, Robinson, and Gault (1946), p. 9-18;

Rossman (1963b), P. K49; Mackevett and others (1967),

Red Mountain: Mackevett and others (1967), p. 97

Adams Inlet: Smith (1942), p. 178
$\mathrm{Cu}, \mathrm{qb}, \mathrm{Mo}, \mathrm{Zn}$

$\mathrm{Co}, \mathrm{Cu}, \mathrm{Ni}$

$\mathrm{Cr}, \mathrm{N}, \mathrm{Ni}$

Commodity $1 /, 2 /$

$\mathrm{Cu}$, Mo

Sb, Ag

Fe

$\mathrm{Co}, \mathrm{Cu}, \mathrm{N} 1$

W

Mo

Bi, Mo, Ag

$\mathrm{Cu}, \mathrm{Au}, \mathrm{Pb}, \mathrm{Mo}, \mathrm{Ag}$,

$\mathrm{Cu}, \mathrm{Mo}$

$\mathrm{Sb}, \mathrm{Au}, \mathrm{Ag}, \mathrm{Zn}$

MO

Co, Fe, Sn, Zn

$\mathrm{Cu}, \mathrm{Au}, \mathrm{Mo}, \mathrm{Ag}, \mathrm{Zn}$

$\mathrm{Cu}, \mathrm{Au}, \mathrm{Mo}, \mathrm{Ag}$

$\mathrm{Cd}, \mathrm{Pb}, \mathrm{Ag}, \mathrm{Zn}$

Mo

Symbols - Sb, antimony; Bi, bismuth; Cd, cadmium; Co, cobalt; Cu, copper; Au, gold; Fe, iron; $\mathrm{Pb}$, lead; Mo, nolybdenum; N1, nickel; Ag, silver; Sn, tin; $\mathrm{W}$, tungsten; $\mathrm{Zn}$, zinc.

.2/ Symbol underlined indicated recorded production. 
Number Name and principal reference(s)

57 Charpentier Inlet: Mackevett and others (1967), p. 151 Mo

58 Gelkie Inlet: Mackevett and others (1967), p. 151 Mo

59 Shag Cove: Mackevete and others (1967), p. 85

60 Francis Island: Buddington (1926), p, 56; Mackevett and others (1967), p. 74-75, 135

61 Willoughby Island: Rossman (1963b), P. X48; Mackevett and others (1967), p. 72-73

62

63

64

65

66

67

68

69

70

71

72

73

74

75

76

l/
Willoughby Island: Mackevett and others (1967), P. 72-73 Cu

North Marble Is land: Rossman (1963b), P. K51; Mackevett and others (1967), P. 70

South Marble Island: Mackevett and others (1967), p. 7

Geikie Inlet: Smith (1942), P. 178

Brady Glacier: Mackevett and others (1967), p. 132

Brady Glacier: Rossman (1963b), P. K50; Mackevett and others (1967), P. 118

Dundas Bay, West Arm: Mackevett and others (1967), p. 85

Unnamed occurrence: Mackevett and others (1967), p. 118

Dundas Bay: Mackevert and others (1967), P. 79

Dundas Bay: Mackevett and others (1967), P. 79

Unnamed occurrence: Mackevett and others (1967), p. 97

Alaska Chief: Mackevett and others (1967), p. 76-78, 135

Dundas Bay: Mackevett and others (1967), p. 128

Whitney: Smith (1942), p. 176-177

Willoughby Cove: Smith (1942), p. 177
Symbols - Sb, antimony; Bi, bismuth; Co, cobalt, Cu, copper; Au, gold; Fe,
Comodity $1 /$

$\mathrm{Co}, \mathrm{Cu}, \mathrm{Ag}, \mathrm{Zn}$

$\mathrm{Sb}, \mathrm{BI}, \mathrm{Cu}, \mathrm{Au}, \mathrm{Mn}$, Ni, $\mathrm{Ag}, \mathrm{Zn}$

$\mathrm{Sb}, \mathrm{Cu}, \mathrm{Au}, \mathrm{Pb}, \mathrm{Ag}$

$\mathrm{Cu}, \mathrm{Fe}, \mathrm{Zn}_{\mathrm{n}}$

$\mathrm{Cu}, \mathrm{NI}$

Mo

$\mathrm{Cu}, \mathrm{Fe}$

$\mathrm{Au}$

$\mathrm{Cu}$

Au

$\mathrm{Cu}, \mathrm{Pb}, \mathrm{Mo}, \mathrm{Ag}$

$\mathrm{Cu}, \mathrm{Mo}$

$\mathrm{Bi}, \mathrm{Pb}, \mathrm{Mo}, \mathrm{Ag}, \mathrm{Zn}$

$\mathrm{Bi}, \mathrm{Co}, \mathrm{Cu}, \mathrm{Au}, \mathrm{Mn}$, Mo, $\mathrm{Ni}, \mathrm{Ag}, \mathrm{Zn}$

$\mathrm{Fe}$

Mo

$\mathrm{Cu}, \mathrm{Mo}$ iron; Pb, lead; Mn, manganese; Mo, molybdenum; Ni, nickel; Ag, silver; $\mathrm{n}$, zinc. 


$\begin{array}{lll}\text { Number } & \text { Name and princlpal reference } & \text { Cormodi } \\ 77 & \text { Marvitz: Rossman }(1959 b), p .211 & \text { Au } \\ 78 & \text { Yakobi Island: Overbeck }(1919), \text { p. } 121 & \text { Cu, Au } \\ \text { l/ Symbols - Cu, copper; Au, gold. } & \\ \text { 2/ Symbol underlined indicates recorded production. }\end{array}$

\section{PLACER DEPOSITS}

Number Name and principal reference(s)

Cormodity $\underline{1}$ '

79-80 Lituya Bay: Mertie (1933), p. 133-136; Rossman (1957), 10 p.; Thomas and Berryhill (1962), p. 37-39

Gold, platinum

81 Oregon King Consolidated: Mackevett and others (1967), P. 120

Gold

82

Brady Glacier: Rossman (1963b), p. K50-K5L

Gold

83

Dundas River: Mackevett and others (1967), P. 120

Gold

84

Dundas River: Rossman (1963b), P. KSO

Gold

If Gold has been produced from most of the listed placers.

\section{REFERENCES}

Buddington, A. F., 1926, Mineral investigations in southeastern Alaska: U.S. Geol. Survey Bul1, 783, P. 41-62.

Kennedy, G. C., and Walton, M. S., 1946, Geology and associated mineral deposits of some ultrabasic rock bodies in southeastern Alaska: U.S. Geol. Survey Bu11. 947-D, p. 65-84.

Mackevett, E. M., Jr., Brew, D. A., Hawley, C. C., Huff, L. C., and Smith, J. G., 1967, Mineral resources of Glacier Bay National Monument, Alaska: U.S. Geol. Survey open-file rept., $176 \mathrm{p}$.

Mertie, J. B., Jr., 1933, Notes on the geography and geology of Lituya Bay: U.S. Geol. Survey Bull. 836, p. 117-135.

overbeck, R. M., 1919, Geology and mineral resources of the west coast of Chichagof Island: U.S. Geol. Survey Bul1. 692, P.91-136.

Rossman, D. L., 1957, Ilmenite-bearing beach sands near Lituya Bay, Alaska: U.S. Geol. Survey open-file rept., $10 \mathrm{p}$.

----, 1959a, Geology and ore deposits of the Reid Inlet area, Glacier Bay, Alaska: U.S. Geol. Survey Bu11. 1058-8, p. 33-59. 
Rossman, D. L., 1959b, Geology and ore deposits of northwestern Chichagof Island, Alaska: U.S. Geol. Survey Bull, 1058-E, p. 139-216.

-..., 1963a, Geology and petrology of two stocks of layered gabbro in the Fairweather Range, Alaska: U.S. Geol. Survey Bull. 1121-F, P. F1-F50.

.---., 1963b, Geology of the eastern part of the Mount Fairweather quadrangle, Glacier Bay, Alaska: U.S. Geol. Survey Bull. 1I2l-K, p. Kl-K57.

Smith, P. S., 1942, Occurrences of molybdenum minerals in Alaska: U.S. Geol, Survey Bu11. 926-C, P. 161-207.

Thomas, B. I., and Berryhi11, R, V., 1962, Reconnaissance studies of Alaskan beach sands, eastern Gulf of Alaska: U.S. Bur. Mines Rept. Inv. 5986, 40 p.

Twenhofel, W. S., Reed, J. C., and Gates, G. O., 1949, Sore mineral investigations in southeastern Alaska: U.S. Geol. Suney Bull. 963-A, P. 1-45,

Twenhofel, W. S., Robinson, G. D., and Gault, H. R., 1946, Molybdenite Investigations in sout heastern Alaska: U.S. Geol. Survey Bu11. 947-B, p. 7-38.

\section{SOURCES OF DATA ON DISTRIBUTION OF IGNEOUS ROCKS}

Loney, R. A., Berg, H. C., Pomeroy, J. S., and Brew, D. A., 1963, Reconnaissance geologic map of Chichagof Is land and northwestern Baranof Island, Alaska: U,S Geol. Survey Misc. Geol. Inv. Map I-388.

Mackevett, E. M., Jr., Brew, D. A., Hawley, C. C., Huff, L. C., and Smith, J. G., 1967, Mlaeral resources of Glacier Bay National Monument, Alaska: U.S. Geol. Survey open-file rept., fig. $3 B$.

Rossman, D. L., 1959, Geology and ore deposits of northwestern Chichagof Island, Alaska: U.S. Geol. Survey Bull. 1058-E, p1. L2.

-.--, 1963, Geology of the eastern part of the Mount Fairweather quadrangle, Glacier Bay, Alaska: U.S. Geol. Survey Bull. 112l-K, pl. 1. 


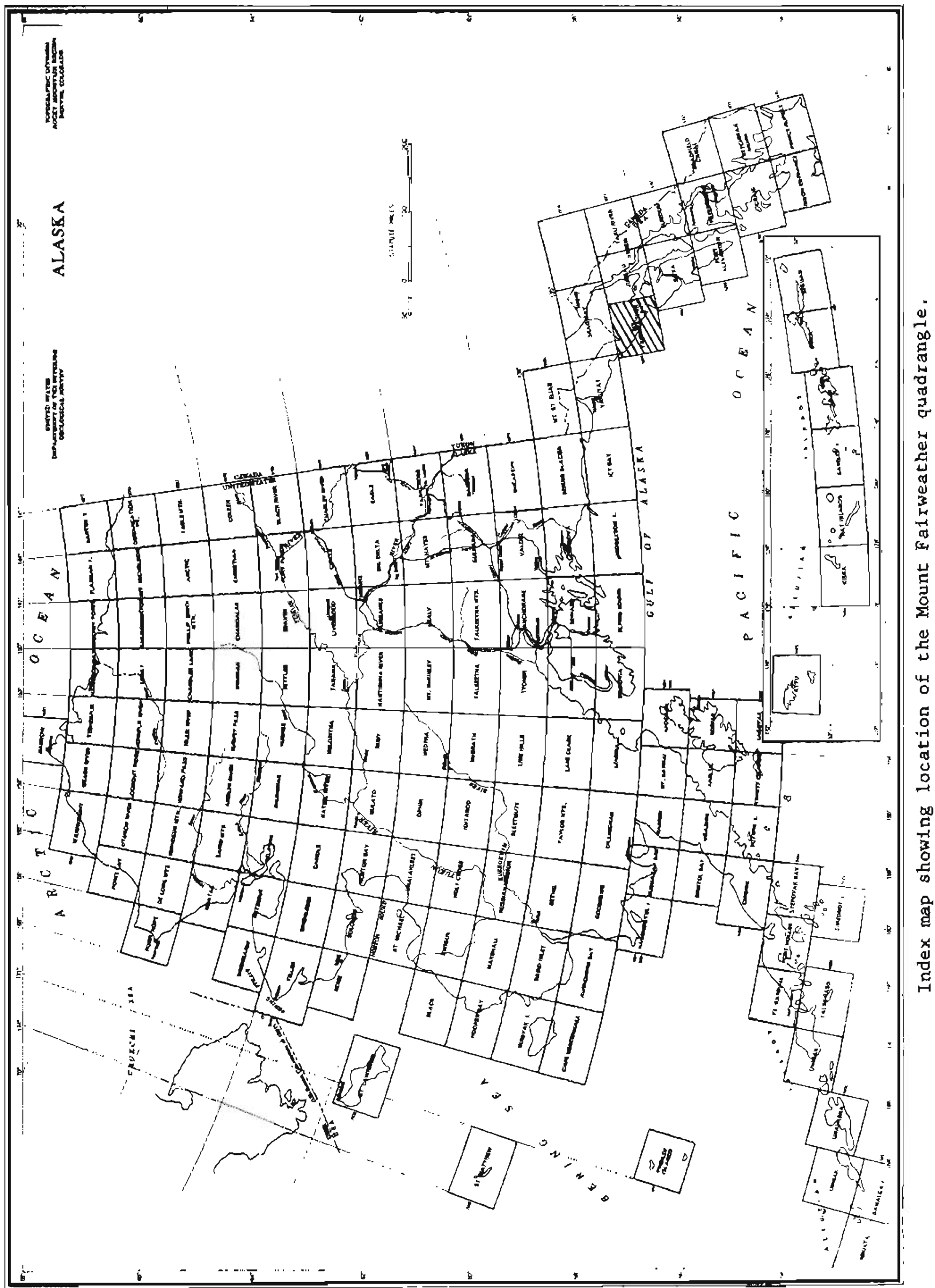

